\title{
Linking Binge Drinking, Depression to SES? An age old question with fresh eyes
}

\author{
Reka Sundaram-Stukel ${ }^{\star 2,1}$, Benjamin Wiseman² and Anne L. Ziege ${ }^{2}$ \\ 'Department of Agriculture and Applied Economics, University of Wisconsin-Madison, Madison, WI, USA; ${ }^{2}$ Wisconsin State \\ Department of Public Health, Madison, WI, USA
}

\section{Objective}

Our primary goal is to move towards establishing a causal link between binge drinking, mental health, employment and income.

\section{Introduction}

One of the key questions in health economics is what is the direction of causality: does poverty cause poor health outcomes; does low education cause poor health outcomes; does poor health result in lack of productivity; does poor health cause poor educational and income outcomes; and how is this all related to mental health if at all. We are used to breaking down data into fragments as researchers: an investigator who is predominantly focused on health outcomes will approach the problem with disease as the dependent variable and income as the conditioning variable. However, if we are interested in income inequality we will reverse the direction and income will be the dependent variable with health status as the conditioning variable.

The representation above allows us to visualize data as a function of multiple fragments. For example if we want to understand how depression is related to income, one can look at the figure to observe that with lower income there is a higher likelihood of being depressed. With this simple illustration we can see that establishing causal links can be very tricky, if not incredibly challenging.

\section{Methods}

Two methods are: applied descriptive analysis and estimation. We approach this without causality in mind, but with an intention to explore how behavior responds to income, education, labor and health. Our descriptive approach looks at trends in binge drinking and mental health as it affects key economic outcomes such as education, employment, and income. For each outcome we then run a simple probit model controlling for a variety of characteristics. The key co-variates in these models are income, employment and health.

It is very useful to look at these simple probits because often it is hard to separate the effects of income on health, employment on income, health on employment, education on employment, health and income, and finally income, employment, health and education on mental health and substance abuse.

\section{Results}

Our estimated results are rather interesting. Examining the marginal probits, e.g. figures 1.3 , and 1.5 , we show that there isn't a significant income effect, nor do we find significant education or employment effects associated with binge drinking. In fact we find that in Wisconsin binge drinking is a health burden for those who are eligible to drink irrespective of education and that the effect is significant; we also find that higher levels of education increase the probability of being unemployed but not significantly. The second set of probit estimates, e.g. figure 1.7, show that poor health is indeed associated with outcomes lower employment as compared to other groups, and higher probability of depression. The last set of probits, e.g. figure 1.1, show that retired, self employed and employed are less likely to be depressed but not significantly so, and those who are unable to work have a higher estimated probablilty to be depressed.
Income doesn't appear to have a significant estimated effect on depression.

\section{Conclusions}

Our analysis provide insights into the question of socio-economic status (SES), binge drinking, and depression in three important ways. First, we explore the relationship between SES and binge drinking and we find that binge drinking is SES invariant. Second we find that depression is not associated with income it does have a strong relationship with employment status. We are in the process of unpacking the effects of SES, binge drinking and depression to move beyond associational inferences to causal inferences.
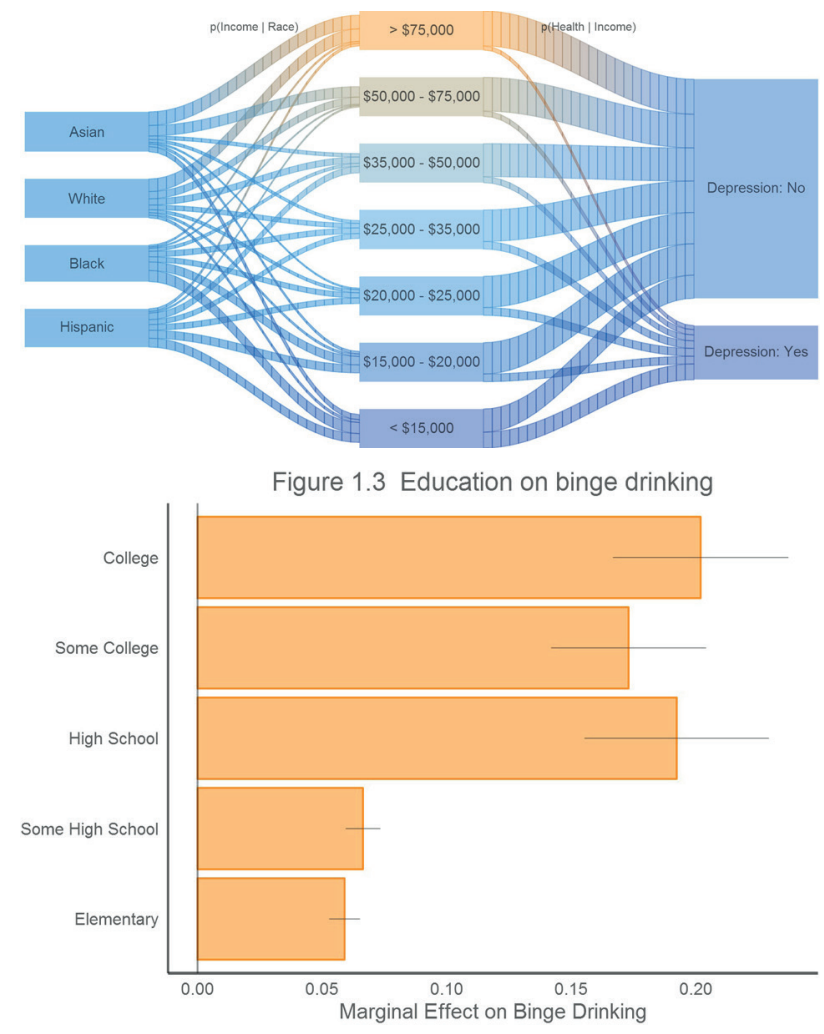
ISDS 2016 Conference Abstracts

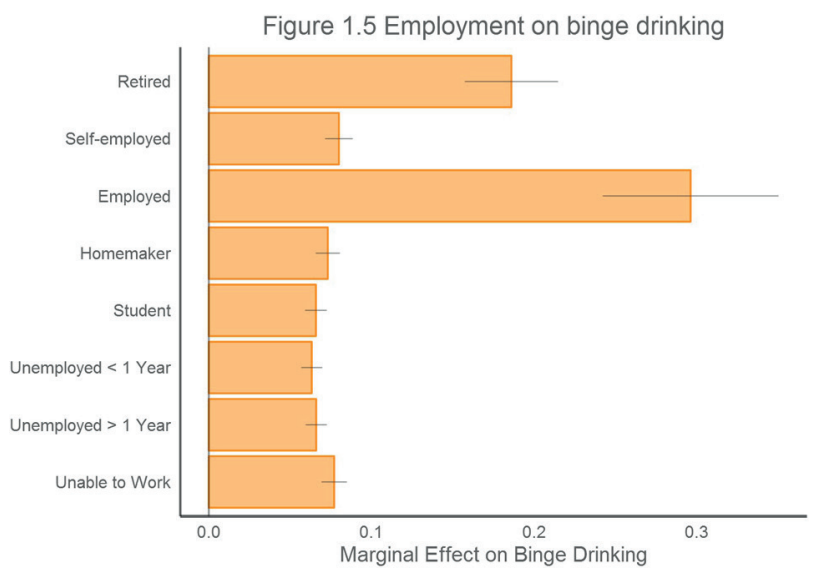

Figure 1.7 General health on employment
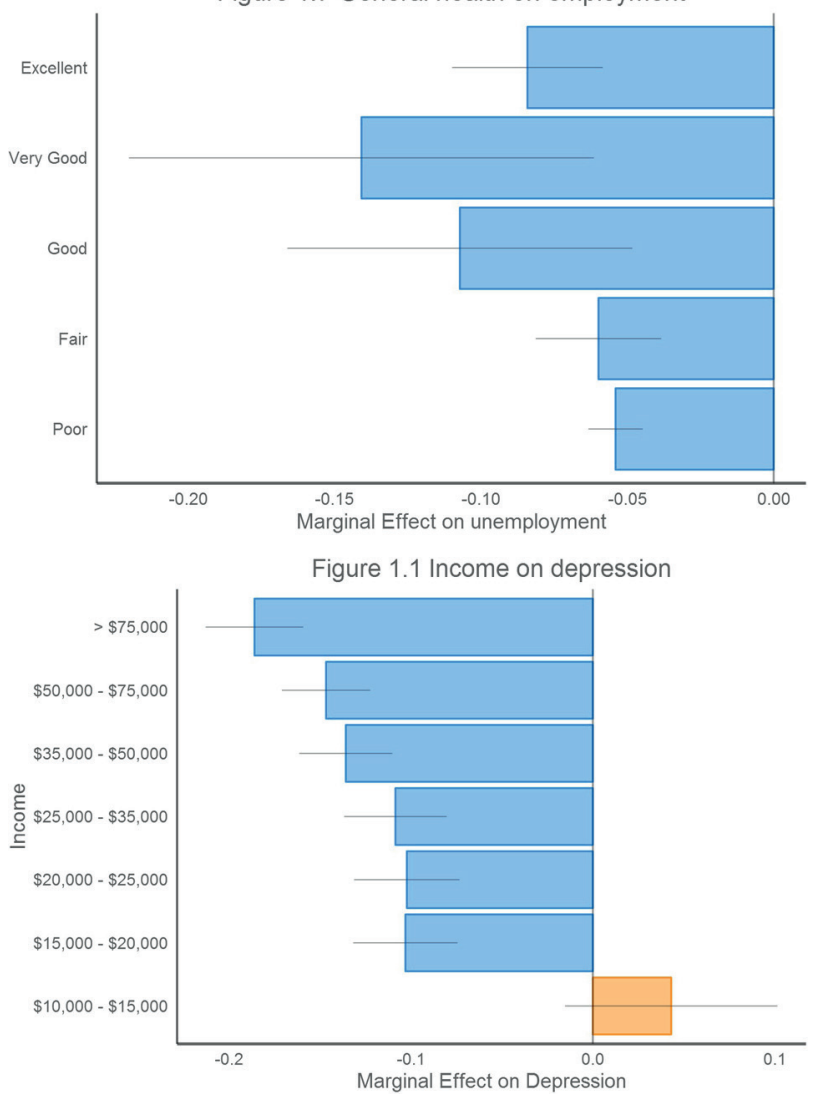

\section{Keywords}

Binge Drinking; Depression; Employment; Income; Education

\section{Acknowledgments}

We acknowledge the staff at the office of health informatics for many useful comments.

\section{References}

Cawley John, and Ruhm Christopher J. (2012). "The Economics of Risky Health Behaviors." Handbook of Health Economics, Vol. 2, pages 95-199.

*Reka Sundaram-Stukel

E-mail: Reka.SundaramStukel@dhs.wisconsin.gov 